\title{
Feasibility and safety of robot-assisted thoracic surgery for lung lobectomy in patients with non-small cell lung cancer: a systematic review and meta-analysis
}

\author{
Shiyou Wei ${ }^{1,3+}$, Minghao Chen ${ }^{2 \dagger}$, Nan Chen ${ }^{1,3}$ and Lunxu Liu ${ }^{1,3^{*}}$
}

\begin{abstract}
Background: The aim of this study is to evaluate the feasibility and safety of robot-assisted thoracic surgery (RATS) lobectomy versus video-assisted thoracic surgery (VATS) for lobectomy in patients with non-small cell lung cancer (NSCLC).

Methods: An electronic search of six electronic databases was performed to identify relevant comparative studies. Meta-analysis was performed by pooling the results of reported incidence of overall morbidity, mortality, prolonged air leak, arrhythmia, and pneumonia between RATS and VATS lobectomy. Subgroup analysis was also conducted based on matched and unmatched cohort studies, if possible. Relative risks (RR) with their 95\% confidence intervals (Cl) were calculated by means of Revman version 5.3.
\end{abstract}

Results: Twelve retrospective cohort studies were included, with a total of 60,959 patients. RATS lobectomy significantly reduced the mortality rate when compared with VATS lobectomy $(R R=0.54,95 \% \mathrm{Cl} 0.38-0.77 ; P=0.0006)$, but this was not consistent with the pooled result of six matched studies ( $R R=0.12,95 \% \mathrm{Cl} 0.01-1.07 ; P=0.06)$. There was no significant difference in morbidity between the two approaches ( $R R=0.97,95 \% \mathrm{Cl} 0.85-1.12 ; P=0.70$ ).

Conclusions: RATS lobectomy is a feasible and safe technique and can achieve an equivalent short-term surgical efficacy when compared with VATS, but its cost effectiveness also should be taken into consideration.

Keywords: Robot-assisted thoracic surgery, Video-assisted thoracic surgery, Minimally invasive surgery, Lung lobectomy

\section{Background}

Lobectomy is considered to be the standard therapy for patients with non-small cell lung cancer (NSCLC) at an early stage, and a minimally invasive approach such as video-assisted thoracic surgery (VATS), rather than thoracotomy, has been recommended to this group of patients [1]. Since the initial VATS lobectomy described in the early 1990s [2, 3], growing evidence has suggested that VATS is an appropriate approach, which shows

\footnotetext{
*Correspondence: lunxu_liu@aliyun.com

${ }^{\dagger}$ Equal contributors

'Department of Thoracic surgery, West China Hospital, Sichuan University, No. 37, Guoxue Alley, Chengdu, Sichuan 610041, China

${ }^{3}$ Western China Collaborative Innovation Center for Early Diagnosis and Multidisciplinary Therapy of Lung Cancer, Sichuan University, No. 37, Guoxue Alley, Chengdu, Sichuan 610041, China

Full list of author information is available at the end of the article
}

superior perioperative outcomes and improved long-term survival for selected patients with early stage NSCLC when compared with conventional thoracotomy [4, 5]. Despite such demonstrated advantages of VATS, some shortcomings such as steep learning curve, difficult handeye coordination, lack of instrument flexibility, and twodimensional vision might still restrict the development of this technique [6, 7].

Robot-assisted thoracic surgery (RATS) is a relatively new technique for minimally invasive lung lobectomy. And the initial feasibility and safety of RATS lobectomy have been described by several publications in the past 10 years [8-11]. RATS lobectomy appears to present some advantages of VATS approach in terms of decreased blood loss, less impairment in pulmonary function, and short hospital length of stay when compared to 
conventional thoracotomy [12-14]. However, RATS lobectomy may be limited by its potential longer operative time and higher hospital costs. Ignoring these disadvantages, advocates still emphasize the benefits of RATS in regard to three-dimensional high-definition view, improved ergonomics less steep learning curve, and better maneuverability of instruments $[15,16]$. Unfortunately, there is lack of evidence-based information on the feasibility and safety of RATS lobectomy in patients with NSCLC and whether RATS lobectomy can achieve equivalent short-term surgical efficacy when compared with VATS is also unknown. Therefore, we conducted this systematic review and meta-analysis of published studies in an attempt to assess the feasibility and safety of RATS lobectomy versus those with VATS.

\section{Materials and methods}

\section{Search strategy}

Electronic searches were performed in PubMed, Embase, Web of Science, Cochrane Central Register of Controlled Trials, Cochrane Database of Systematic Reviews, and ClinicalTrials.gov up to December 2016 without language restriction. We combined the terms "VATS OR video-assisted thoracic surgery OR thoracoscopic surgery" and "robotics OR robot OR robotic surgery OR computer-assisted surgery OR da Vinci" to search for eligible comparative studies. References of included studies were also manually searched to identify potentially relevant studies.

\section{Study selection}

Preferred reporting items for systematic reviews and meta-analyses (PRISMA) flow chart was adapted to depict the study selection process [17]. After removing duplicates, two reviewers (SYW and MHC) independently reviewed the relevant studies by checking the titles, abstracts, and full-texts. Studies were eligible for inclusion in this meta-analysis if they were randomized or nonrandomized controlled trials comparing RATS to VATS. We excluded studies which were relevant to RATS wedge resection or segmentectomy and those which did not contain a comparative group. In the case of duplicate publications with accumulating numbers of patients or increased lengths of follow-up, we only included the most recent or complete reports for our analysis.

\section{Data extraction}

Two reviewers (SYW and MHC) independently examined the included studies and extracted data points pertaining to first author's name, year of publication, study design, study period, surgical technique for RATS or VATS lobectomy, preoperative patient demographics (number of patients, geographic location, lobe distribution, and pathological stage), intraoperative parameters (operative time, blood loss, and conversion), and postoperative parameters (dissected lymph nodes station and number, hospital length of stay, prolonged air leak, arrhythmia, pneumonia, composite morbidity, perioperative mortality, and costs). The primary outcomes were perioperative mortality and morbidity, and the secondary outcomes were operative time, blood loss, hospital length of stay, prolonged air leak, arrhythmia, pneumonia, conversion, dissected lymph nodes (LNs) station and number, and costs. Discrepancies were resolved by group discussion and consensus with a senior investigator (LXL).

\section{Quality assessment}

The risk of bias for each included observational study was assessed using the Newcastle-Ottawa Scale (NOS). The NOS includes three parts for cohort studies: selection (four scores assigned), comparability (two scores assigned), and outcome (three scores assigned). Studies with scores of 0 to 3,4 to 6 , and 7 to 9 were considered to be low, moderate, and high quality, respectively.

\section{Statistical analysis}

Meta-analysis was performed by pooling the results of reported incidence of overall morbidity, mortality, prolonged air leak, arrhythmia, and pneumonia. Subgroup analysis was also conducted based on matched and unmatched cohort studies, if possible. Relative risks (RR) and their 95\% confidence intervals (CI) were calculated for discontinuous data. Summary RRs were calculated by using fixed-effect models when heterogeneity among studies was considered to be statistically insignificant. Otherwise, random-effect models were used to combine the results. Heterogeneity among the studies was identified by conducting a standard Cochrane's Q test with a significance level of $\alpha=0.10$. The $I^{2}$ statistic test was performed to further examine heterogeneity. $I^{2} \geq 50 \%$ was considered to indicate substantial heterogeneity. Besides, visual inspection of the funnel plots was used to identify potential publication bias. All $P$ values were two-tailed, and $P<0.05$ was considered to be statistically significant. All analysis was conducted with Review Manager Version 5.3 (Cochrane Collaboration, Software Update, Oxford, United Kingdom, 2014).

\section{Results}

\section{Literature search}

The initial search identified 1007 references. After duplicates were removed, 791 articles were retrieved for title and abstract assessment, and 21 articles were selected for full-text evaluation. Nine articles were excluded; of which, three articles were duplicate publications and six articles were relevant to RATS segmentectomy or wedge resection. Finally, a total of 12 retrospective cohort 
studies were included in this systematic review and meta-analysis [18-29]. The flow chart of selection for included studies is depicted in Fig. 1.

\section{Characteristics and risk of bias assessment}

The included studies were published from 2010 to 2017. Of the 12 studies, seven studies [21, 23-26, 28, 29] were conducted in North America, two [22, 27] in Europe, two $[18,19]$ in Asia, and one [20] in Australia. Overall, 60,959 patients were identified for the analysis; of whom, 4727 patients underwent RATS and 56,232 patients received VATS lobectomy. The average age across various studies ranged from 26 to 88 years old. Of the 12 included studies, eight [18-22, 26-28] referred to the surgical technique of RATS, seven [18-21, 26-28] reported the arms of the da Vinci surgical system, and only five $[18,21,26-28]$ provided information about the type of da Vinci surgical system.

The quality of the included studies assessed by the NOS was generally acceptable, with a mean NOS scores of 6.8 (standard deviation $=0.7$ ). For most included studies, the methodological quality in terms of cohort selection and comparability was adequate. However, the follow-up periods were limited for most studies except for two studies $[26,28]$. The characteristics and risk of bias assessment of the included studies were shown in Table 1

\section{Assessment of perioperative outcomes}

A total of ten studies [18, 20-24, 26-29] that compared RATS to VATS lobectomy reported perioperative mortality outcome, including six matched studies $[18,20,22$, $23,27,28]$ and four unmatched studies [21, 24, 26, 29]. Mortality was $0.6 \%(29 / 4521)$ and $1.3 \%(720 / 55,560)$ for patients undergoing RATS and VATS, respectively. The pooled analysis of mortality demonstrated that when compared to VATS lobectomy, RATS showed a significantly lower mortality $(\mathrm{RR}=0.54,95 \%$ CI $0.38-0.77 ; P$ $=0.0006$; fixed model), and this result was in line with the pooled result of three unmatched studies $(R R=0.58$, 95\% CI $0.40-0.84 ; P=0.003)$, but was not consistent with the pooled result of six matched studies $(R R=0.12$, 95\% CI $0.01-1.07 ; P=0.06$ ) (Fig. 2). There was no statistical heterogeneity among the studies $\left(I^{2}=0 \%, P=0.83\right)$. Visual inspection of the funnel plots did not identify a potential publication bias.

Composite morbidity was reported in nine studies [18-20, 23-28] (seven matched studies [18-20, 23, 25, $27,28]$ and two unmatched studies $[24,26])$. The overall morbidity rate was $46.5 \%(1652 / 3552)$ and $45.1 \%$ $(17,759 / 39,403)$ in patients who underwent RATS and VATS lobectomy, respectively. The result of metaanalysis revealed that there was no statistically significant difference in composite morbidity between RATS and VATS lobectomy $(\mathrm{RR}=0.97,95 \%$ CI $0.85-1.12 ; P=$ 0.70; random model), and there was a significant heterogeneity among the eight studies $\left(I^{2}=62 \%, P=0.006\right)$ (Fig. 3). Publication bias was not evident from visual inspection of the funnel plots.

Prolonged air leak was reported in six studies [20-22, 27-29], and the incidence of prolonged air leak was 9.8\% (157/1596) and 9.5\% (1641/17,219) for patients undergoing VATS and RATS, respectively. The incidence of arrhythmia that was reported in five studies [20-22, $28,29]$ was $10.4 \%(163 / 1568)$ for RATS lobectomy and 9.7\% $(1667 / 17,191)$ for VATS lobectomy. Five studies $[21,22,25,28,29]$ reported the data on the incidence of pneumonia, which was 3.6\% (66/1837) and 3.3\% (582/ $17,460)$ for patients undergoing RATS and VATS lobectomy, respectively. Five studies [18, 20, 26-28] provided the rate of conversion, and the incidence of conversion was $7.8 \%(25 / 319)$ and $5.6 \%(22 / 393)$ for RATS and

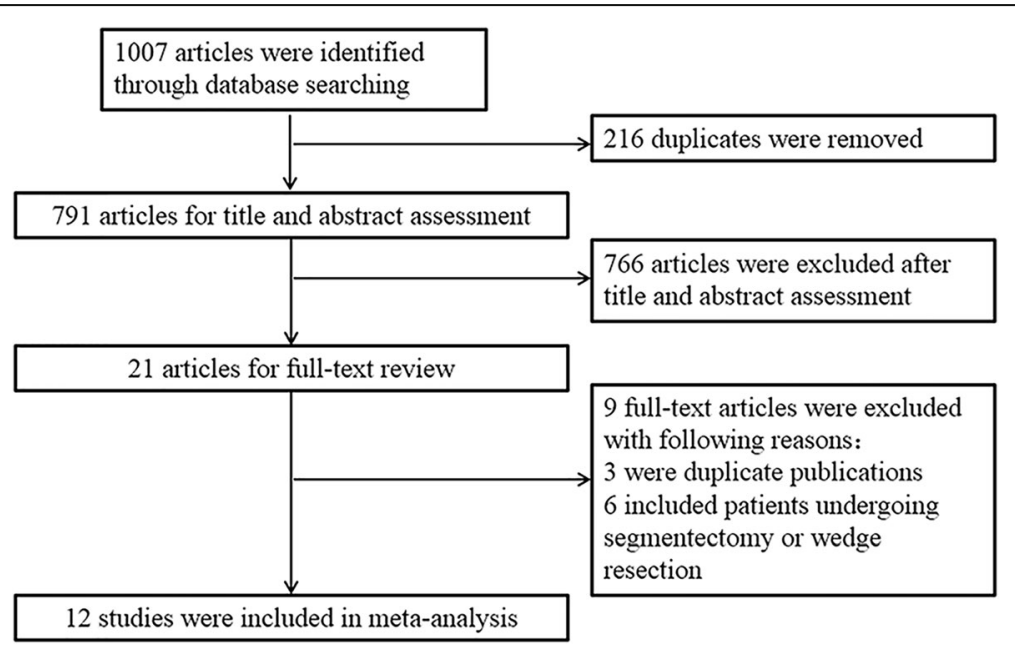

Fig. 1 The PRISMA flow chart of selection for included studies 


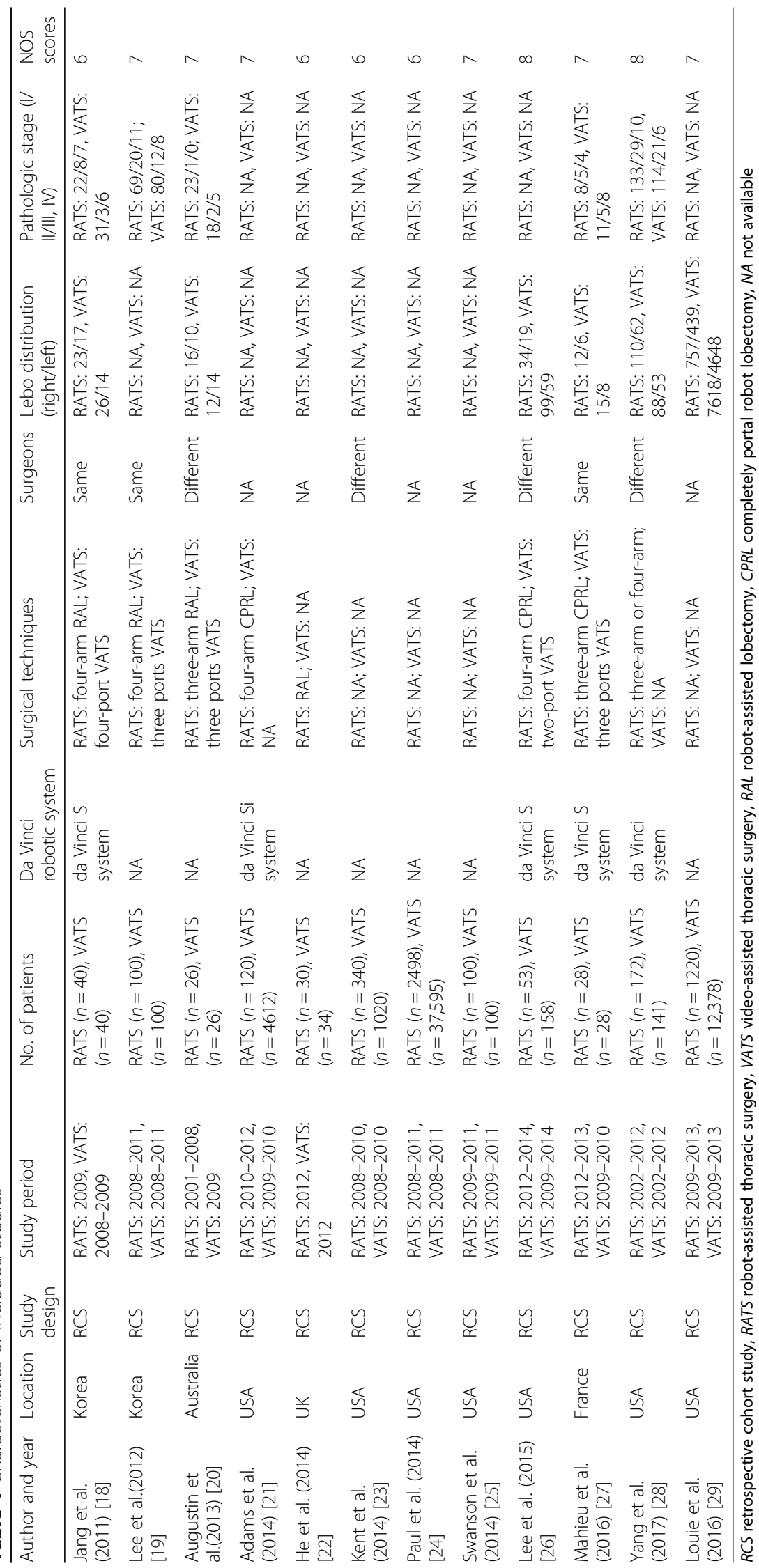




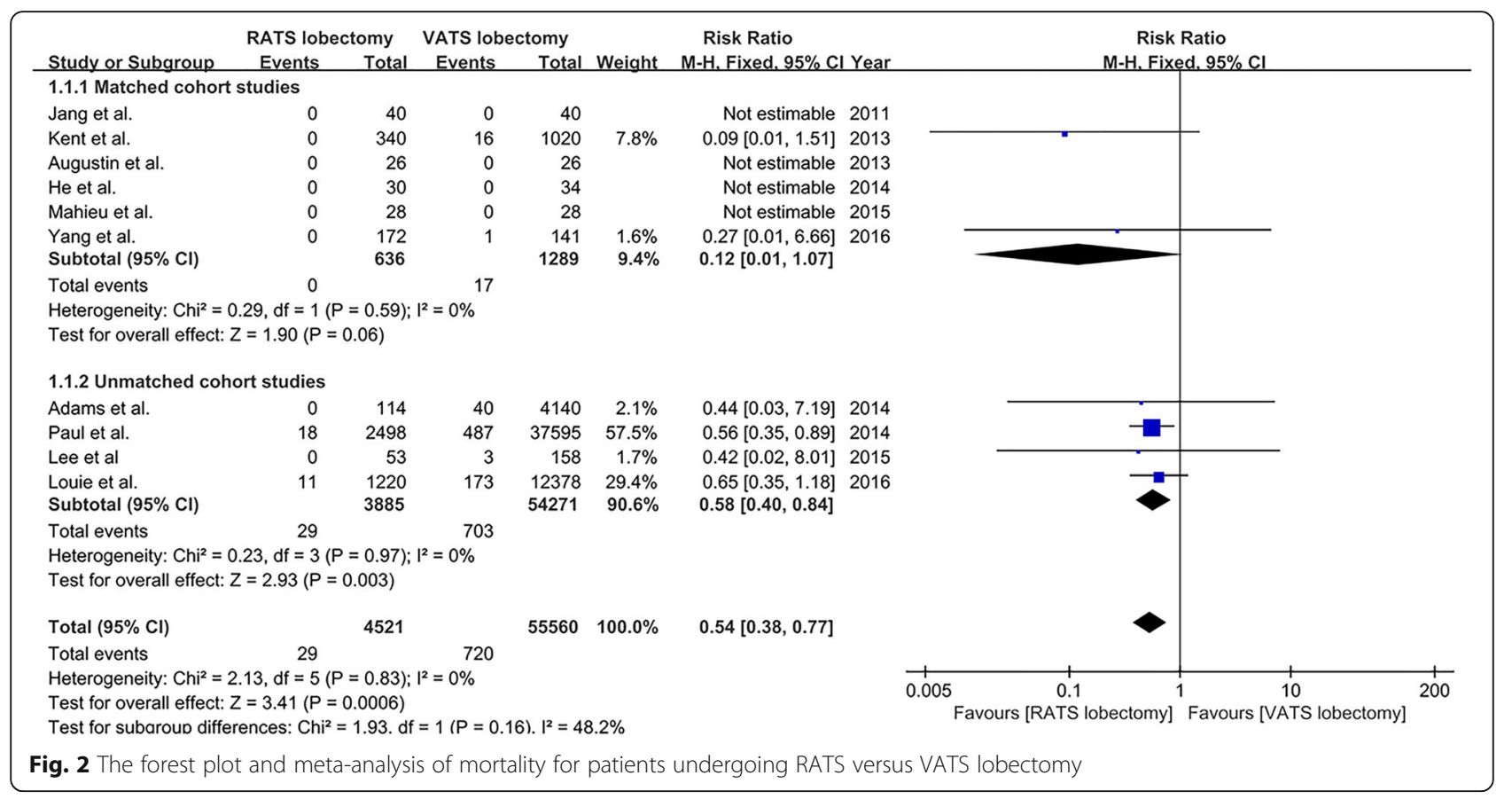

VATS lobectomy, respectively. The meta-analysis on prolonged air leak, arrhythmia, pneumonia, and conversion all showed no significant differences between RATS and VATS lobectomy (prolonged air leak RR $=1.01,95 \%$ CI 0.86-1.19, $P=0.92$; arrhythmia $R R=1.05,95 \% \mathrm{CI}$ $0.89-1.23, P=0.57$; pneumonia $\mathrm{RR}=0.79$, 95\% CI $0.60-$ 1.04, $P=0.09$; conversion $\mathrm{RR}=1.17,95 \%$ CI $0.66-2.07$, $P=0.58$; fixed model) (Fig. 4).
For the 12 studies [18-29] that compared RATS to VATS lobectomy, operative time was significantly longer in RATS group in six studies [18-21, 26, 29], shorter in one study [22], no difference in two studies [25, 27], and not reported in three studies [23, 24, 28]. No significant difference was found in blood loss between RATS and VATS lobectomy in two studies [18, 27]. Only one study [19] showed a significant shorter hospital length of stay

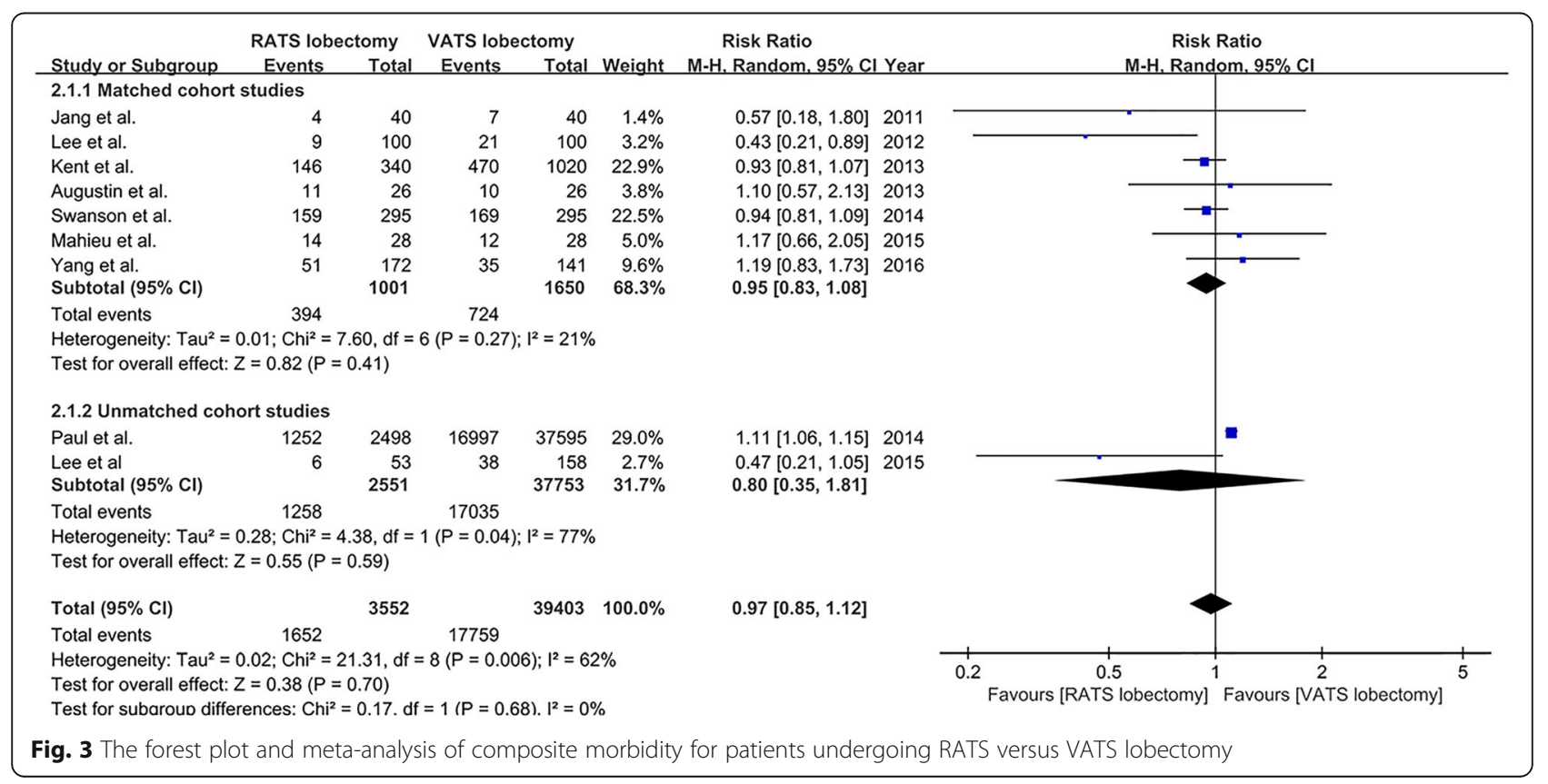




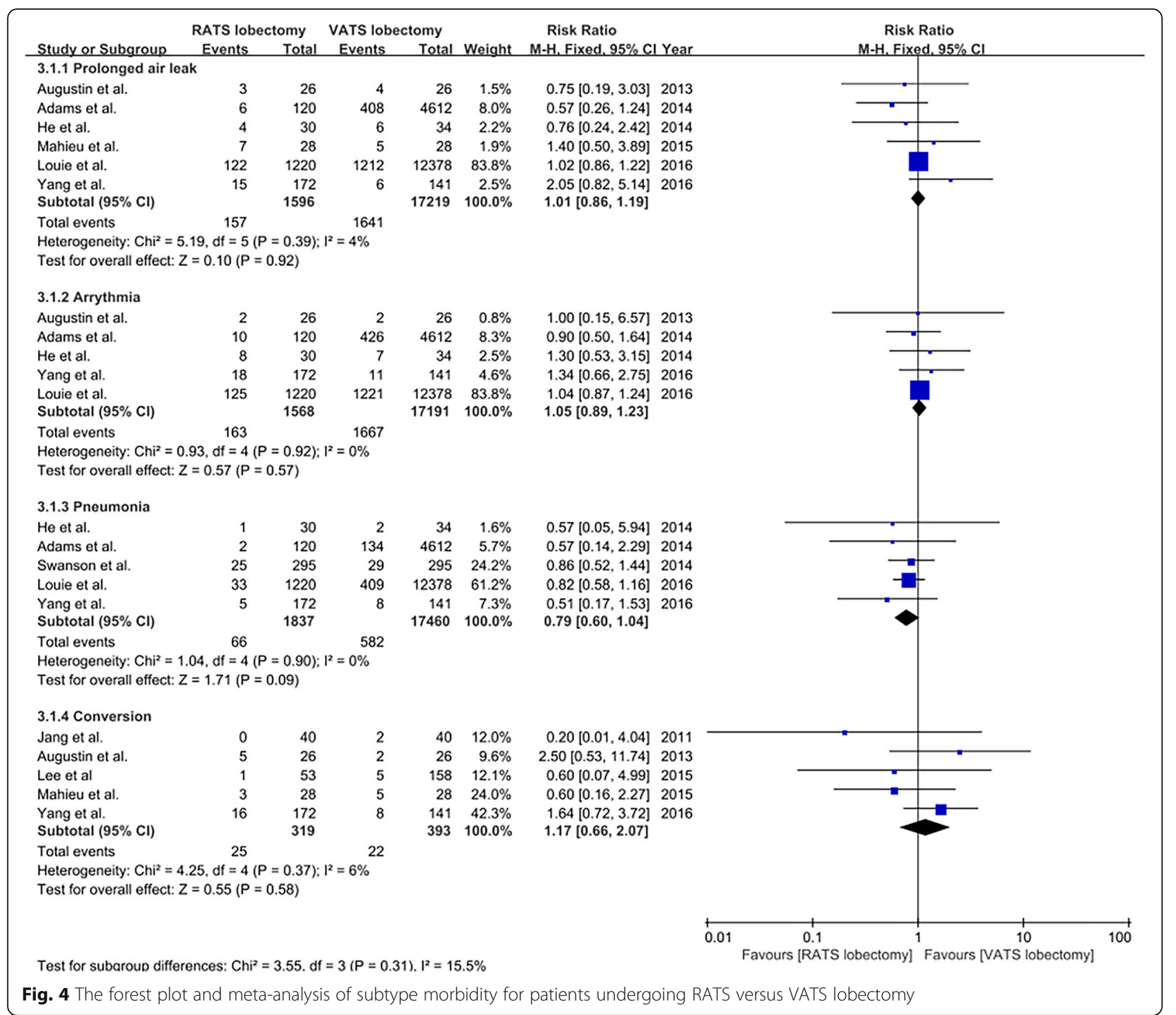

when comparing RATS to VATS lobectomy, and ten studies [18, 20, 21, 23-29] did not observed a difference between the two approaches. The number of dissected lymph nodes station ranged from five to seven and the number of removed lymph nodes ranged from 14 to 24 for RATS lobectomy, which were comparable to VATS lobectomy. However, costs were significantly increased for RATS lobectomy in the included studies (Table 2).

\section{Discussion}

Since the first use of the da Vinci robotic surgical system for pulmonary lobectomy which was reported in 2002 [30], several studies $[8,9]$ have showed the feasibility and safety of this novel technique for lobectomy. A systematic review performed by Cao et al. [31] showed that perioperative mortality for patients who underwent pulmonary resection by RATS ranged from 1 to $3.8 \%$ and overall morbidity ranged from 10 to $39 \%$. However, Cao et al. did not conduct a pooled analysis to assess the safety and efficacy of RATS lobectomy compared to those of VATS lobectomy. In another study [32], eight retrospective observational studies were eligible for meta-analysis and were evaluated for perioperative morbidity and mortality, but the meta-analysis included patients who underwent lobectomy, segmentectomy, and wedge resection.

The present systematic review and meta-analysis identified twelve retrospective cohort studies, including a total of 60,959 patients who underwent RATS lobectomy $(n=4727)$ and VATS lobectomy $(n=56,232)$. The metaanalysis revealed that RATS lobectomy significantly reduced the mortality rate when compared with VATS lobectomy $(\mathrm{RR}=0.54,95 \% \mathrm{CI} 0.38-0.77 ; P=0.0006$ ), but this was not consistent with the pooled result of six matched studies ( $\mathrm{RR}=0.12,95 \% \mathrm{CI} 0.01-1.07 ; P=0.06$ ). This result could be explained in part by the highly 
Table 2 Perioperative outcomes of included studies

\begin{tabular}{|c|c|c|c|c|c|c|c|c|c|c|c|c|}
\hline \multirow[t]{2}{*}{ Study } & \multicolumn{2}{|c|}{ Operative time (min) } & \multicolumn{2}{|c|}{$\underline{\text { Blood loss (ml) }}$} & \multicolumn{2}{|c|}{ Hospital length of stay (d) } & \multicolumn{2}{|c|}{ Dissected LNs station } & \multicolumn{2}{|c|}{ Dissected LNs number } & \multicolumn{2}{|l|}{ Costs } \\
\hline & RATS & VATS & RATS & VATS & RATS & VATS & RATS & VATS & RATS & VATS & RATS & VATS \\
\hline Jang et al. & 240 & $161^{*}$ & 219 & 245 & 6 & 7 & 7 & 8 & 22 & $26^{*}$ & NA & NA \\
\hline Lee et al. (2012) & 209 & $157^{*}$ & NA & NA & $6.3^{*}$ & 8.9 & 7.3 & 6.0 & 24.8 & 23.6 & NA & NA \\
\hline Augustin et al. & 215 & $183^{*}$ & NA & NA & 11 & 9 & NA & NA & NA & NA & $2507 €$ & $1736 €$ \\
\hline Adams et al. & 241 & $179.8^{*}$ & NA & NA & 4.7 & 5.3 & 4.1 & NA & 9.4 & NA & NA & NA \\
\hline He et al. & $145.50^{*}$ & 162.79 & NA & NA & NA & NA & NA & NA & NA & NA & NA & NA \\
\hline Kent et al. & NA & NA & NA & NA & 5.9 & 5.7 & NA & NA & NA & NA & NA & NA \\
\hline Paul et al. & NA & NA & NA & NA & 5 & 5 & NA & NA & NA & NA & $\$ 22,582$ & $\$ 17,874^{*}$ \\
\hline Swanson et al. & 269.4 & 253.8 & NA & NA & 6.07 & 5.83 & NA & NA & NA & NA & $\$ 25,041$ & $\$ 20,477^{*}$ \\
\hline Lee et al. (2015) & 161 & $123^{*}$ & NA & NA & 3 & 3 & NA & NA & $17^{*}$ & 11 & NA & NA \\
\hline Mahieu et al. & 190 & 185 & 100 & 200 & 6 & 7 & 5 & 3 & 14 & 14 & NA & NA \\
\hline Yang et al. & NA & NA & NA & NA & 4 & 4 & $5^{*}$ & 3 & NA & NA & NA & NA \\
\hline Louie et al. & 186 & $173^{*}$ & NA & NA & 4 & 4 & NA & NA & NA & NA & NA & NA \\
\hline
\end{tabular}

${ }^{*} P<0.05$

RATS robot-assisted thoracic surgery, VATS video-assisted thoracic surgery, LNs lymph nodes, NA not available

selected patients at the beginning of this surgical technique; therefore, this result should be interpreted with caution. Moreover, the overall perioperative morbidity rate of RATS was similar to that of VATS lobectomy, and no statistically significant differences were observed in the incidence of postoperative prolonged air leak, arrhythmia, and pneumonia when comparing RATS to VATS lobectomy. Anyway, these outcomes suggest that RATS lobectomy is a safe and feasible surgical approach for patients with lung cancer and can achieve an equivalent short-term surgical efficacy compared with VATS lobectomy.

With respect to the operative results, most included studies reported a longer operative time for RATS compared to VATS lobectomy [18-21, 26, 29]. This can be explained by several potential factors. First, the knowledge and experience of RATS lobectomy for surgeons were inadequate at the beginning of the learning curve and most included studies just reported their initial attempts to RATS lobectomy. Second, prolonged operative time was reported to be caused by setting up the robotic system [18, 20]. Third, different surgery approaches might lead to different operative time. As reported in the study of Augustin et al. [20], the overall operative time was longer in the RATS group, but when comparing the anterior approach of RATS to VATS lobectomy, there was no significant difference on operative time. But it should be mentioned that the increased operative time in robotic surgery did not seem to have a negative impact on postoperative results, since there was no increase in short-term morbidity and mortality for patients. Besides, operative time for RATS approach has been shown to significantly shorten after the initial learning period. Therefore, with the increased knowledge and experience of RATS, operative time for RATS would be comparable to VATS.

In addition, in our present study, higher costs for lung lobectomy with the da Vinci surgical system was observed in most included studies. In a large case series, Park et al. [33] demonstrated that RATS lobectomy was on average \$3981 more expensive than VATS lobectomy, but $\$ 3988$ cheaper than open lobectomy. And the increased costs of RATS compared with VATS lobectomy occurred primarily in the first hospital day, which could be explained as the additional disposable costs of the robotic instruments and a higher percentage of additional procedural costs. Augustin et al. [20] also indicated that two thirds of the additional costs for RATS lobectomy were caused by disposables and the use of robotic instruments. However, in a retrospective analysis of 176 RATS lobectomies compared to 76 VATS lobectomies, Dylewski et al. [34] showed that direct costs was reduced by $\$ 560$ per case in RATS group. And the majority of costs saving benefited from reduced length of hospital stay and lower overall nursing care costs. In addition, according to Deen et al. [35], shortening operative time, eradicating unnecessary laboratory work, reducing respiratory therapy, and minimizing stays in the intensive care unit would contribute to a decrease of hospital costs for patients who underwent RATS lobectomy. However, the costs associated with the overall substantial acquisition and maintenance for the robotic system was usually not included in analysis in most studies. In fact, a da Vinci robotic surgical system currently costs between $\$ 1$ and $\$ 2.5$ million in the United States [36], and is associated with annual maintenance costs of approximately $\$ 100,000$ to $\$ 170,000[33,37]$. Therefore, in the Japanese health care system, it is necessary to 
perform at least 300 robotic operations per year in each institution to avoid financial deficit with the current process of robotic surgical system management [38]. Since the effectiveness of RATS lobectomy is equivalent with increased costs when compared with that of VATS procedure, manufacturers of robotic surgical system would reduce supply costs by developing new generation robotic system to be more competitive.

There are several limitations existing in the present systematic review and meta-analysis. Firstly, it should be acknowledged that the data included in the present meta-analysis were extrapolated from 12 retrospective cohort studies. Although the heterogeneity was negligible among the included studies, selection bias of retrospective studies may lead to unbalanced selection of patients. Secondly, the characteristics of included patients and the surgical techniques were not clearly described in some included studies, which may lead a bias for the meta-analysis results. Thirdly, specific criteria for the definition of outcomes, such as prolonged air leak, conversion, and morbidity, were not clearly stated in most included studies. Fourthly, there are lacks of longterm follow-up outcomes for the comparison of RATS lobectomy with VATS lobectomy. Hence, future studies should emphasize the rigorous eligibility criteria, clear definition of outcomes, and long-term follow-up data.

\section{Conclusions}

In conclusion, the current systematic and meta-analysis demonstrates that RATS lobectomy is a feasible and safe technique for selected patients and can achieve an equivalent short-term surgical efficacy when compared with VATS procedure. However, longer operative time and cost effectiveness of RATS should be taken into consideration, and long-term oncological efficacy of the RATS approach remains to be seen.

\section{Abbreviations \\ Cl: Confidence interval; CPRL: Completely portal robot lobectomy; LNs: Lymph nodes; NA: Not available; NOS: Newcastle-Ottawa Scale; NSCLC: Non-small cell lung cancer; RAL: Robot-assisted lobectomy; RATS: Robot-assisted thoracic surgery; RCS: Retrospective cohort study; RR: Relative risks; VATS: Video-assisted thoracic surgery.}

\section{Acknowledgements}

None.

\section{Funding}

None.

\section{Availability of data and materials}

All data generated or analyzed during this study are included in this published article.

\section{Authors' contributions}

SYW, MHC, and LXL contributed to the study conception and design. SYW and MHC performed the collection of data. SYW, MHC, and NC conducted the data analysis and interpretation. All authors contributed to the manuscript writing. All authors read and approved the final manuscript.

\section{Competing interests}

The authors declare that they have no competing interests.

\section{Consent for publication}

None.

Ethics approval and consent to participate None.

\section{Publisher's Note}

Springer Nature remains neutral with regard to jurisdictional claims in published maps and institutional affiliations.

\section{Author details}

1Department of Thoracic surgery, West China Hospital, Sichuan University, No. 37, Guoxue Alley, Chengdu, Sichuan 610041, China. ${ }^{2}$ Center for Reproductive Medicine, Guangdong Women and Children Hospital, Guangzhou 510010, China. ${ }^{3}$ Western China Collaborative Innovation Center for Early Diagnosis and Multidisciplinary Therapy of Lung Cancer, Sichuan University, No. 37, Guoxue Alley, Chengdu, Sichuan 610041, China.

Received: 10 December 2016 Accepted: 2 May 2017

Published online: 08 May 2017

\section{References}

1. Detterbeck FC, Lewis SZ, Diekemper R, et al. Executive summary: diagnosis and management of lung cancer, 3rd ed: American College of Chest Physicians evidence-based clinical practice guidelines. Chest. 2013;143:7S-375.

2. Kirby TJ, Mack MJ, Landreneau RJ, et al. Initial experience with videoassisted thoracoscopic lobectomy. Ann Thorac Surg. 1993;56:1248-52. discussion 52-3.

3. Walker WS, Carnochan FM, Pugh GC. Thoracoscopic pulmonary lobectomy. Early operative experience and preliminary clinical results. J Thorac Cardiovasc Surg. 1993;106:1111-7.

4. Yan TD, Black D, Bannon PG, et al. Systematic review and meta-analysis of randomized and nonrandomized trials on safety and efficacy of videoassisted thoracic surgery lobectomy for early-stage non-small-cell lung cancer. J Clin Oncol. 2009;27:2553-62.

5. Cao C, Manganas C, Ang SC, et al. A meta-analysis of unmatched and matched patients comparing video-assisted thoracoscopic lobectomy and conventional open lobectomy. Ann Cardiothorac Surg. 2012;1:16-23.

6. Petersen RH, Hansen HJ. Learning curve associated with VATS lobectomy. Ann Cardiothorac Surg. 2012;1:47-50.

7. Arad T, Levi-Faber D, Nir RR, et al. The learning curve of video-assisted thoracoscopic surgery (VATS) for lung lobectomy - a single Israeli center experience. Harefuah. 2012:151:261-5. 320.

8. Park BJ, Flores RM, Rusch WW. Robotic assistance for video-assisted thoracic surgical lobectomy: technique and initial results. J Thorac Cardiovasc Surg. 2006;131:54-9.

9. Gharagozloo F, Margolis M, Tempesta B, et al. Robot-assisted lobectomy for early-stage lung cancer: report of 100 consecutive cases. Ann Thorac Surg. 2009;88:380-4.

10. Augustin F, Bodner J, Wykypiel H, et al. Initial experience with robotic lung lobectomy: report of two different approaches. Surg Endosc. 2011;25:10813 .

11. Kumar A, Asaf BB, Cerfolio RJ, et al. Robotic lobectomy: the first Indian report. J Minim Access Surg. 2015;11:94-8.

12. Veronesi G, Galetta D, Maisonneuve P, et al. Four-arm robotic lobectomy for the treatment of early-stage lung cancer. J Thorac Cardiovasc Surg. 2010; 140:19-25.

13. Cerfolio RJ, Bryant AS, Skylizard L, et al. Initial consecutive experience of completely portal robotic pulmonary resection with $4 \mathrm{arms}$. I Thorac Cardiovasc Surg. 2011;142:740-6.

14. Oh DS, Cho I, Karamian B, et al. Early adoption of robotic pulmonary lobectomy: feasibility and initial outcomes. Am Surg. 2013;79:1075-80

15. Melfi FM, Fanucchi O, Davini F, et al. VATS-based approach for robotic lobectomy. Thorac Surg Clin. 2014;24:143-9. v.

16. Veronesi G. Robotic thoracic surgery: technical considerations and learning curve for pulmonary resection. Thorac Surg Clin. 2014;24:135-41. v. 
17. Liberati A, Altman DG, Tetzlaff J, et al. The PRISMA statement for reporting systematic reviews and meta-analyses of studies that evaluate healthcare interventions: explanation and elaboration. BMJ. 2009;339:b2700.

18. Jang HJ, Lee HS, Park SY, et al. Comparison of the early robot-assisted lobectomy experience to video-assisted thoracic surgery lobectomy for lung cancer: a single-institution case series matching study. Innovations (Phila). 2011;6:305-10

19. Lee HS, Jang HJ. Thoracoscopic mediastinal lymph node dissection for lung cancer. Semin Thorac Cardiovasc Surg. 2012;24:131-41.

20. Augustin $\mathrm{F}$, Bodner J, Maier $\mathrm{H}$, et al. Robotic-assisted minimally invasive vs. thoracoscopic lung lobectomy: comparison of perioperative results in a learning curve setting. Langenbecks Arch Surg. 2013;398:895-901.

21. Adams RD, Bolton WD, Stephenson JE, et al. Initial multicenter community robotic lobectomy experience: comparisons to a national database. Ann Thorac Surg. 2014:97:1893-8. discussion 1899-1900.

22. He Y, Coonar A, Gelvez-Zapata S, et al. Evaluation of a robot-assisted videoassisted thoracoscopic surgery programme. Exp Ther Med. 2014;7:873-6.

23. Kent $M$, Wang $T$, Whyte $R$, et al. Open, video-assisted thoracic surgery, and robotic lobectomy: review of a national database. Ann Thorac Surg. 2014;97: 236-42. discussion 242-4.

24. Paul S, Jalbert J, Isaacs AJ, et al. Comparative effectiveness of roboticassisted vs thoracoscopic lobectomy. Chest. 2014;146:1505-12.

25. Swanson SJ, Miller DL, McKenna Jr RJ, et al. Comparing robot-assisted thoracic surgical lobectomy with conventional video-assisted thoracic surgical lobectomy and wedge resection: results from a multihospital database (Premier). J Thorac Cardiovasc Surg. 2014;147:929-37.

26. Lee BE, Shapiro M, Rutledge JR, et al. Nodal upstaging in robotic and video assisted thoracic surgery lobectomy for clinical No lung cancer. Ann Thorac Surg. 2015;100:229-33. discussion 233-4.

27. Mahieu J, Rinieri P, Bubenheim M, et al. Robot-assisted thoracoscopic surgery versus video-assisted thoracoscopic surgery for lung lobectomy: can a robotic approach improve short-term outcomes and operative safety? Thorac Cardiovasc Surg. 2016;4:354-62.

28. Yang HX, Woo KM, Sima CS, et al. Long-term survival based on the surgical approach to lobectomy for clinical stage I nonsmall cell lung cancer: comparison of robotic, video-assisted thoracic surgery, and thoracotomy lobectomy. Ann Surg. 2017:265:431-7.

29. Louie BE, Wilson JL, Kim S, et al. Comparison of video-assisted thoracoscopic surgery and robotic approaches for clinical stage I and stage II non-small cell lung cancer using the Society of Thoracic Surgeons Database. Ann Thorac Surg. 2016;102:917-24.

30. Melfi FM, Menconi GF, Mariani AM, et al. Early experience with robotic technology for thoracoscopic surgery. Eur J Cardiothorac Surg. 2002;21:864-8.

31. Cao C, Manganas C, Ang SC, et al. A systematic review and meta-analysis on pulmonary resections by robotic video-assisted thoracic surgery. Ann Cardiothorac Surg. 2012;1:3-10.

32. Ye X, Xie L, Chen G, et al. Robotic thoracic surgery versus video-assisted thoracic surgery for lung cancer: a meta-analysis. Interact Cardiovasc Thorac Surg. 2015;21:409-14.

33. Park BJ, Flores RM. Cost comparison of robotic, video-assisted thoracic surgery and thoracotomy approaches to pulmonary lobectomy. Thorac Surg Clin. 2008;18:297-300. vii.

34. Dylewski MR, Lazzaro RS. Robotics - the answer to the Achilles' heel of VATS pulmonary resection. Chin J Cancer Res. 2012;24:259-60.

35. Deen $\mathrm{SA}$, Wilson $\mathrm{JL}$, Wilshire $\mathrm{CL}$, et al. Defining the cost of care for lobectomy and segmentectomy: a comparison of open, video-assisted thoracoscopic, and robotic approaches. Ann Thorac Surg. 2014;97:1000-7.

36. Turchetti G, Palla I, Pierotti F, et al. Economic evaluation of da Vinci-assisted robotic surgery: a systematic review. Surg Endosc. 2012;26:598-606.

37. Wei B, D'Amico TA. Thoracoscopic versus robotic approaches: advantages and disadvantages. Thorac Surg Clin. 2014:24:177-88. vi.

38. Kajiwara N, Patrick Barron J, Kato Y, et al. Cost-benefit performance of robotic surgery compared with video-assisted thoracoscopic surgery under the Japanese National Health Insurance System. Ann Thorac Cardiovasc Surg. 2014;21:95-101.

\section{Submit your next manuscript to BioMed Central and we will help you at every step:}

- We accept pre-submission inquiries

- Our selector tool helps you to find the most relevant journal

- We provide round the clock customer support

- Convenient online submission

- Thorough peer review

- Inclusion in PubMed and all major indexing services

- Maximum visibility for your research

Submit your manuscript at www.biomedcentral.com/submit
Biomed Central 